\title{
Carcinoma of the oesophagus and fundus
}

\author{
D. A. W. EDWARDS* \\ M.A., M.D., F.R.C.P.

\section{Medical Research Council Gastroenterology Unit, Central Middlesex Hospital, and Department of Clincial Research*}

\begin{abstract}
Summary
The problems of earlier diagnosis, the diagnostic symptom patterns and radiographic features of carcinoma of the oesophagus and fundus are discussed.
\end{abstract}

\section{Earlier diagnosis}

The probability of cure is low in both these conditions, but the quality of the remaining life could be much improved by earlier diagnosis and initiation of treatment when the patient is still well nourished and dysphagia is not severe. My remarks are based on a study of 180 squamous cancers, and ninety adenocarcinomata causing dysphagia at the oesophago-gastric junction.

Firstly all dysphagia for solids, regardless of age, should be considered neoplastic until proved otherwise by symptom pattern and radiology, and secondly spasm is not a definable entity and should not be accepted as a diagnosis. Oesophagoscopy and biopsy may show carcinoma but a negative report does not exclude it and about $50 \%$ of our first examinations fail to identify neoplastic tissue when it is there. One cannot use hospital consultations for determining the incidence of diseases but our experience at Central Middlesex is that the number of new patients a year with dysphagia from neoplastic causes is about the same as the number of obstructive dysphagias from benign lesions. Careful attention to the details of symptoms and immediate appropriate radiology are the most sensitive indicators of the lesion. The purpose of endoscopy is to obtain a biopsy, because the appearance itself may not distinguish clearly between benign and malignant lesions. The purpose of biopsy is to detect cancer and determine the cell type because the surgeon would not operate or would perform a different operation if the lesion was benign, and the value of radiotherapy and surgery depend upon the cell type. But I want to emphasize again that biopsy cannot exclude carcinoma because a negative biopsy has such a high probability of being wrong. Exfoliative cytology by brush biopsy or lavage might increase

* Medical Research Council Department of Clinical Research, University College Hospital Medical School, London. the probability of obtaining neoplastic cells if they are present. On a probability basis, therefore, the site of the lesion is more helpful in determining the cell type than biopsy. Squamous cancer develops from squamous epithelium above the squamocolumnar junction which is usually within the sphincter segment. Adenocarcinoma develops from the columnar cells below the junction or in the fundus of the stomach.

\section{Symptom patterns}

Dysphagia usually develops at some stage of cancer of the oesophagus but may not be the presenting symptom or sign. This account assumes that dysphagia is present. The symptom patterns follow the mechanical situations which can easily be seen by radiology.

Squamous cancer starts as a nodule which grows around and up and down the lumen. The normal part of the circumference of the wall is easily distensible and a bolus will pass the neoplastic tissue without provoking a sensation until about twothirds of the circumference has been stiffened by the tumour. A large bolus then causes pain or discomfort as it passes and as the tumour grows the discomfort increases or the patient is more and more careful about what he swallows. Commonly dysphagia is noticed at the biggest or lumpiest meal of the week, occurs several times during the next week and within another week is present at every meal unless the patient takes care. From this moment the dysphagia for solids progressively worsens but at this stage there is no dysphagia for liquids provided food has not stuck. A drink may push the bolus through but within days or weeks a drink worsens the problem and is regurgitated, food will not pass at all unless it is liquidized and pain on impact continues, but there is no pain at any other time. As the dysphagia for solids becomes absolute, weight loss begins. Heartburn if present is coincidental and the oesophageal epithelium is not hypersensitive to acid, fruit juices, hot drinks, or alcohol. Inflammatory peptic strictures do not progress as fast as this, have a hypersensitive mucosa, and a drink will push the bolus through for months or years. 
Within days, weeks, and occasionally months, the total dysphagia to solids progresses to dysphagia for liquids so that a cup of tea has to be sipped very slowly. Regurgitation of a mouthful to a cupful of thick saliva becomes frequent and almost total obstruction may develop in a few days. This is because the rate of flow of fluid through a narrow pipe is proportional to the fourth power of the radius. As the radius of the lumen gets smaller a very small reduction has a considerable effect on the rate of flow. Unfortunately this is the stage at which many patients are admitted as an emergency to hospital, either because the G.P. has not taken the dysphagia seriously or because the patient has been on an outpatient or inpatient waiting list for weeks, or is waiting for an X-ray. We should be able to make the diagnosis weeks earlier and act upon the early dysphagia as an emergency.

Adenocarcinoma may present in one of three ways. If the tumour starts in the fundus and extends to the oesophago-gastric junction there may be a large or only a small mass of tumour in the stomach before dysphagia begins. There is no sense of impact or bolus obstruction if the dysphagia occurs because the stomach cannot accommodate much food because of the mass of tumour or because of linitis plastica, but when tumour encroaches on the oesophago-gastric junction there is a sensation that solids are sticking about 5-10 sec after they are swallowed. Commonly at every meal the first and second mouthful sticks with pain lasting a few seconds, and the bolus may be helped on with a drink. After that the rest of the meal may be eaten without discomfort. This self-dilatation may continue for months to a year or longer without other symptoms or weight loss, then there is a sudden, rapid progression of dysphagia for solids then liquids over a period of days or weeks. Again there is no hypersensitivity to acid, heat or alcohol. The second pattern is that dysphagia for lumps of bread and meat suddenly develops and persists without much progression for weeks or months and softer or small pieces of food pass without difficulty until again there is a sudden deterioration. The third pattern is of the sudden onset and rapid progression in days or a few weeks of dysphagia for solids and then liquids without any preceding warning of illness. Dysphagia for liquids in carcinoma always follows a period of dysphagia for solids only, whereas in achalasia dysphagia for liquids and solids is coincident.

The sudden onset with persistence, or progression, of a sense of obstruction to food without hypersensitivity to acid, heat or alcohol is very likely to be caused by a neoplasm. The patient may say he has a pain or indigestion in his chest, or that he vomits when he eats, or that he has a spasm in the chest, or simply that he can't eat meat or bread. The precise $\frac{\$}{\infty}$ details of the problem yield the diagnosis often $\stackrel{\varrho}{C}$ before the radiologist detects anything especially if the lesion is in the fundus. Dysphagia for solids merits urgent investigation and the absence of $\overrightarrow{0}$ weight loss or anorexia only means that dysphagia $\frac{}{0}$ is not absolute and carcinomatosis is not advanced. $\frac{\bar{\rho}}{\bar{T}}$

\section{Radiology}

Squamous carcinoma is rarely missed because के dysphagia does not develop until the lesion is $\vec{\circ}$ extensive. If the patient drinks barium continuously $\overrightarrow{\vec{\omega}}$ in the right anterior oblique $10^{\circ}$ head-down position ${ }_{\sigma}^{\omega}$ the upper and lower extent of that part of the lesion $\bar{C}$ which alters the flexibility and contraction of the 3 . wall of the oesophagus should be clearly demon- if strated. Full distension of the oesophagus at, 은 proximal and distal to, the lesion is essential for $\mathbb{N}_{N}^{\infty}$ accurate discrimination from a benign lesion. $N$ Identification of the lesion is easiest in the right $\underset{\omega}{\sim}$ anterior oblique, after which other views may give more detail. Peptic stricture has smooth short shoulders and a smooth, sometimes twisted bore $\rightarrow$ which is rarely less than $4 \mathrm{~mm}$ diameter, compared with the ragged irregular cauliflower excrescence of $\overrightarrow{0}$ carcinoma which may block the lumen to a thin laye which a grain of rice or a piece of scrambled egg w easily block.

Adenocarcinoma closes the oesophago-gastric junction. If there is a free flow of liquid barium through the junction and no proximal evidence of $\frac{0}{\varnothing}$ an obstructing lesion the patient should swallow $\propto$ pieces of bread-soaked barium. Radiology commonly $\overrightarrow{\overrightarrow{0}}$ fails to demonstrate an early fundal carcinoma 3 causing dysphagia because the elasticity of the wall $\rightleftharpoons$ cannot be adequately challenged and the carcinoma in the fundus cannot be demonstrated for technical $\frac{}{2}$ reasons. At this stage an oesophagoscope or gastroscope may pass into the stomach with a minimal $\frac{\sigma}{3}$ sense of resistance and no abnormality may be seen at several attempts. Sometimes the oesophago- $\frac{\delta}{2}$ gastric junction looks normal but the endoscope will not pass, just as the food will not pass, con- 음 firming the diagnosis of carcinoma. Commonly the $\rightarrow$ barium does not flow freely into the stomach when the subject is erect, the oesophagus is somewhat $\bar{N}$ dilated, and achalasia is suspected. The distinction is easily made because if the oesophagus is emptied, a 0 single swallow of barium in the $10^{\circ}$ head-down $\mathrm{\omega}$ position will demonstrate the persisting peristaltic wave in carcinoma and its absence or disorganization in achalasia. The carcinoma makes the wall and $\mathbb{\complement}$ shoulders of the narrow outflow channel rigid and $\stackrel{\infty}{+}$ unaltering in shape and bore, whereas the wall is constantly writhing and changing the shape and bore $\frac{\vec{\Phi}}{\mathbb{D}}$ of the narrowed segment and adjacent wall in $\stackrel{\mathbb{P}}{\mathbb{D}}$ achalasia. Special techniques are necessary to dis- $\stackrel{\unrhd}{\varrho}$ 
tinguish malignant from benign lesions but they are usually successful. The patient, however, is right "more often than the physician who should be right more often than the radiologist who should be right more often than the endoscopist. The patient and his symptom pattern is the most sensitive diagnostic tool; the distinction of cancer from peptic stricture and from achalasia can be readily made by symptoms supported by good radiology.

\section{Diagnostic flow chart}

The symptom patterns described in this symposium have been combined into the following flow chart. The results of a similar method of analysis by symptoms is given in Table 1.

TABle 1. Diagnosis by Algorithm: 242 patients

Predicted \begin{tabular}{lrrrrr}
\hline & \multicolumn{5}{c}{ Final Diagnosis } \\
\hline A & A & B & HH & C \\
B & 28 & 1 & 1 & 0 \\
HH & 1 & 47 & 0 & 3 \\
C & 0 & 0 & 23 & 0 \\
\end{tabular}

Misc. Correct 22; incorrect 4; outside scope 13; otherwise failed 8 . $A=$ achalasia; $B=$ benign stricture; $\mathbf{H H}=$ pseudodysphagia from hiatal hernia; $\mathbf{C}=$ cancer of oesophagus or fundus unspecified.

\section{Dysphagia diagnostic flowchart}

\section{Pharyngeal problems}

Pouch; stricture or web; neuromuscular lesion.

Pouch: food and liquid leave mouth and return immediately or after many hours or if neck is compressed.

Stricture or web: liquid flows easily unless tight stricture, solids impact or returned within $1 \mathrm{sec}$ of swallow.

Neurological or muscular: failure to close nose from pharynx causes nasal regurgitation. Failure to close larynx from pharynx causes immediate cough unless recurrent laryngeal damage.

\section{Questions:}

(1) Food and liquid leave throat and get into gullet without difficulty? Yes $\longrightarrow 2$; no $\longrightarrow 3$.

(2) Does food or liquid return almost instantaneously or after many hours? Yes=pouch; no= oesophageal problem $\longrightarrow 7$.

(3) Is there difficulty making swallow movement? Yes $=$ cortical inhibition, neurological; no $\longrightarrow 4$.

(4) Does liquid leave throat easily without causing cough but solids do not or have to be forced through narrow pipe? Yes = stricture or web; no $\longrightarrow 5$.

(5) Does drinking cause 'nose-trick' or immediate coughing? Yes = muscle or nerve lesion (palatal or epiglotic or pharyngeal failure); no (means delayed cough) $\longrightarrow 6$.
(6) Is dysphonia present? Yes=malignant or idiopathic damage recurrent laryngeal nerve; no= oesophagotracheal fistula or high oesophageal obstruction.

\section{Oesophageal problems}

Benign stricture; cancer of oesophagus; cancer of fundus; achalasia.

Stricture and early cancer: open tube narrowed by rigid process; liquids flow easily; solids impact with pain; no pain except when solids stuck.

Achalasia or diffuse spasm: tube closed by elastic contraction of sphincter but can be opened by pressure. Both liquids and solids slow to move through sphincter. No dramatic impact sensation or pain, commonly spontaneous pain.

\section{Questions:}

(7) Can liquids be drunk easily and quickly as fast as usual except when food has stuck? Yes=open channel to liquid, i.e. benign or malignant stricture or pseudodysphagia or some achalasias $\longrightarrow 8$; no $=$ tube closed to liquids, i.e. achalasia or tight high oesophageal stricture or advanced cancer $\rightarrow 14$.

(8) Does food impact with pain or sometimes stick so that nothing will move on? Yes=stricture (benign or malignant) $\longrightarrow 10$; no (i.e. no impact, no pain) $\longrightarrow 9$.

(9) Does feeling that food is stuck come on during eating? Yes = achalasia; no (i.e. comes on sometime after eating $)=$ pseudodysphagia.

Benign and malignant stricture Distinguished by the following features which give high probability of one diagnosis.
Benign (B)

Intermittent block.

Mucosa often acid, heat and alcohol sensitive.

Slow progression months or years.

\section{Malignant $(M)$}

Continuous and progressive block from one meal.

Usually not sensitive.

Rapid progression days or weeks.
Questions: Ask 10, 11, 12, 13 and combine probabilities.

(10) More than 12 months since first block? Yes $=B ;$ no $=M$.

(11) Can go days without block when eating normally ? Yes $=B$ (probably ring or early peptic stricture); no $=M$ or advanced peptic.

(12) Does hot drink or alcohol or fruit juice burn or sting as it goes down? Yes $=B$; no $=M$.

(13) Did dysphagia progress in weeks or few months almost from one meal? $\mathrm{Yes}=\mathrm{M} ; \mathrm{no}=\mathrm{B}$. 
Achalasia, fundal adenocarcinoma and advanced squamous carcinoma may cause complete or almost complete block to liquids and drinking is very slow. Are distinguished by following features which give high probabilities of one diagnosis.

Achalasia $(A)$

Slow progression in months.

Liquids and solids almost equally difficult from beginning.

No impact pain commonly episodes of spontaneous pain.

Can eat most of meal if taken very slowly or build up pressure in oesophagus.
Questions: Ask 14, 15, 16, 17, 18 and combine probabilities.

(14) Spontaneous chest pain present? Yes=A; no $=C$.

(15) Trouble with liquids rapidly worse in recent weeks? Yes $=\mathrm{C} ;$ no $=\mathrm{A}$.

(16) Period when solids but not liquids difficult? Yes $=C$; no $=A$.

(17) Pain provoked by eating in the beginning, not otherwise present ? Yes $=\mathrm{C}$; no $=A$.

(18) Can eat and drink most of meal if does so very slowly ? $\mathrm{Yes}=\mathrm{A}$ or $\mathrm{C}$; no $=\mathrm{C}$.

The results of the use of this scheme in 242 consecutive patients with dysphagia are shown in Table 1. 